\title{
An Anatomically Placed Tibial Tunnel does not Completely Surround a Simulated PCL Reconstruction Graft in the Proximal Tibia
}

\author{
Christopher Kim, MD ${ }^{1}$ Dustin Baker, MD² Brian Albers, BS ${ }^{3} \quad$ Scott G. Kaar, MD² \\ 1 Private Practice, lowa Clinic, West Des Moines, lowa \\ ${ }^{2}$ Department of Orthopedic Surgery, Saint Louis University, \\ Saint Louis, Missouri \\ ${ }^{3}$ SSM Health Cardinal Glennon Children's Hospital, Saint Louis, \\ Missouri \\ Address for correspondence Scott G. Kaar, MD, Department of \\ Orthopedic Surgery, Saint Louis University, 1008 South Spring \\ Avenue, $1^{\text {st }}$ Floor, SLUCare Academic Pavillion, Saint Louis, MO 63110 \\ (e-mail: scott.kaar@health.slu.edu).
}

J Knee Surg

\begin{abstract}
Introduction It is hypothesized that anatomic tunnel placement will create tunnels with violation of the posterior cortex and subsequently an oblique aperture that is not circumferentially surrounded by bone. In this article, we aimed to characterize posterior cruciate ligament $(\mathrm{PCL})$ tibial tunnel using a three-dimensional (3D) computed tomography (CT) model.

Methods Ten normal knee CTs with the patella, femur, and fibula removed were used. Simulated $11 \mathrm{~mm}$ PCL tibial tunnels were created at 55, 50, 45, and 40 degrees. The morphology of the posterior proximal tibial exit was examined with 3D modeling software. The length of tunnel not circumferentially covered (cortex violation) was measured to where the tibial tunnel became circumferential. The surface area and volume of the cylinder both in contact with the tibial bone and that not in contact with the tibia were determined. The percentages of the stick-out length surface area and volume not in contact with bone were calculated.

Results The mean stick-out length of uncovered graft at 55, 50, 45, and 40 degrees were $26.3,20.5,17.3$, and $12.7 \mathrm{~mm}$, respectively. The mean volume of exposed graft at $55,50,45$, and 40 degrees were $840.8,596.2,425.6$, and $302.9 \mathrm{~mm}^{3}$, respectively. The mean percent of volume of exposed graft at 55, 50, 45, and 40 degrees were 32, 29, 25 , and $24 \%$, respectively. The mean surface of exposed graft at $55,50,45$, and 40 degrees were $372.2,280.4,208.8$, and $153.3 \mathrm{~mm}^{2}$, respectively. The mean percent of surface area of exposed graft at 55, 50, 45, and 40 degrees were $40,39,34$, and $34 \%$, respectively.

\section{Keywords \\ - posterior cruciate ligament \\ - tunnel \\ - reconstruction \\ - computed tomography}

Conclusion Anatomic tibial tunnel creation using standard transtibial PCL reconstruction techniques consistently risks posterior tibial cortex violation and creation of an oblique aperture posteriorly. This risk is decreased with decreasing the angle of the tibial tunnel, though the posterior cortex is still compromised with angles as low as 40 degrees. With posterior cortex violation, a surgeon should be aware that a graft within the tunnel or socket posteriorly may not be fully in contact with bone. This is especially relevant with inlay and socket techniques.
\end{abstract}

received

February 1, 2021

accepted after revision

November 16, 2021 (c) 2022. Thieme. All rights reserved.

Thieme Medical Publishers, Inc., 333 Seventh Avenue, 18th Floor, New York, NY 10001, USA
DOI https://doi.org/

10.1055/s-0041-1741430. ISSN 1538-8506. 
Knee ligament reconstruction involves introduction of grafts into bony sockets and tunnels. There is meaningful research and discussion in optimizing reconstruction outcomes, including graft choices, fixation options, anatomic considerations, and overall reconstruction techniques. In the early postoperative period, the strength of the reconstruction largely depends on the fixation techniques utilized. Ultimately, graft incorporation into the bone plays a more important role in fixation strength. This healing process may take several months and involves remodeling of both the graft's collagen fibers and the surrounding trabecular bone. ${ }^{1}$ In fact, poor tendon to bone healing has been implicated as one of the primary causes of anterior cruciate ligament $(\mathrm{ACL})$ reconstruction failure. ${ }^{2,3}$ There have been numerous studies researching the effect of graft length within the bony tunnels in ACL reconstruction. ${ }^{4-8}$ It is generally accepted that a minimum amount of graft, typically $15 \mathrm{~mm}$, is likely necessary within the bone tunnels to ensure adequate graft incorporation and subsequent fixation strength. Though similar research related to posterior cruciate ligament (PCL) reconstruction is not as abundant, the same principles of optimizing graft incorporation may still apply.

The anatomy of the PCL insertion on the posterior tibia has been extensively reported. ${ }^{9-12}$ In placing anatomic tunnels, one must consider the unique bony architecture of the proximal posterior tibia, including the sloping central depression between the medial and lateral portions of the tibial plateau known as the PCL facet. ${ }^{13}$ This PCL facet is distinct from the tibial plateau and posterior tibial cortex and serves as an important landmark during anatomic tunnel placement. As the bulk of the PCL appears to insert along the posterior aspect of the facet, ${ }^{10}$ it has been recommended that the tibial tunnel be placed just anterior to the so-called "champagne glass drop-off." Due to the shape of the posterior tibia and the desired location of an anatomic tibial tunnel, there has been concern for breaking the posterior cortex during tunnel creation. ${ }^{14}$ This would result in an oblique aperture, and a socket that is not fully surrounding by bone. Lee et al described this possibility, particularly with increasing the angle of the tibial guide. ${ }^{14}$

The current study aimed to better characterize the shape of the tibial tunnel that is created in the posterior tibia using a three-dimensional (3D) computed tomography (CT) model. It is hypothesized that anatomic tunnel placement will create tunnels with violation of the posterior cortex and subsequently an oblique aperture that is not circumferentially surrounded by bone. We sought to utilize CT scans and $3 \mathrm{D}$ reconstruction software to describe the shape of the tunnel and to also determine the change in the amount of posterior cortex violation as the tunnel angle is changed. It is hypothesized that decreasing the tunnel angle would result in less posterior cortex violation. We hypothesized that decreasing the tibial tunnel angles would result in more graft that is in contact with bone as it traverses the tunnel.

\section{Methods}

Institutional review board approval was obtained from our institution for this research study. Ten anonymous patients'

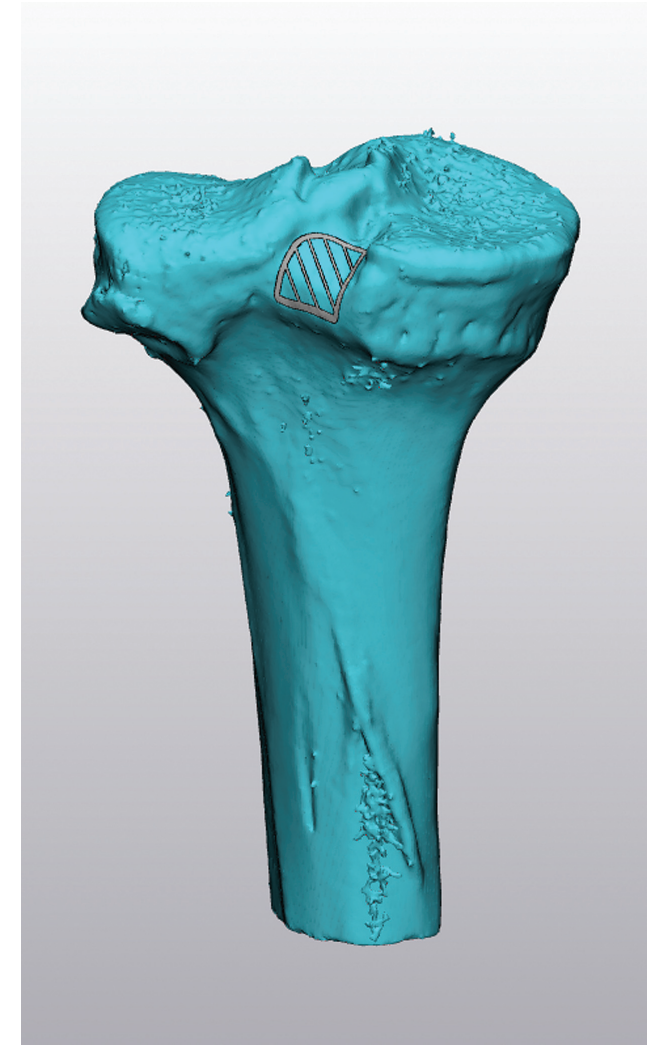

Fig. 1 Representative three-dimensional (3D) computed tomography $(C T)$ with posterior cruciate ligament $(P C L)$ tibial footprint identified (shaded area).

knee CT scans with no tibia bone abnormality were randomly selected from our institution's imaging database. The CT scans were uploaded, the femur and fibula subtracted out, and the tibias were modeled in 3D software (Materialise Mimics 23 and 3-Matic 15; Leuven, Belgium). Simulated $11 \mathrm{~mm}$ tunnels were then placed in each tibia model with an exit point at the posterior proximal tibia situated in the center of the PCL tibial footprint. The center of the PCL tibia footprint was identified on the intercondylar fossa between the tibial plateaus (-Fig. 1). The simulated tunnel exit point was centered between the anterolateral bundle insertion on the superolateral aspect of the fossa (anterolateral slope) and the posteromedial bundle insertion on the inferomedial aspect of the fossa (posteromedial slope) along the posterior aspect of the PCL facet. Four different tibial tunnel drilling angles were simulated at 55, 50, 45, and 40 degrees in relation to a line parallel to the tibial plateau. These drilling angles were felt to represent a reasonable range of what is used for PCL reconstruction tibial drilling, though there are no universally accepted published drilling angle recommendations. The anterior tibial exit points for the tunnels were determined chiefly by the angle of the simulated tibial tunnels and exited the anteromedial tibia just medial to the tibial tubercle.

A simulated cylinder was placed in each tunnel to measure the length of the tunnel from the proximal most aspect as determined by tibial bone removal posteriorly ( - Fig. 2 A and B). The length of tibial tunnel that was not 


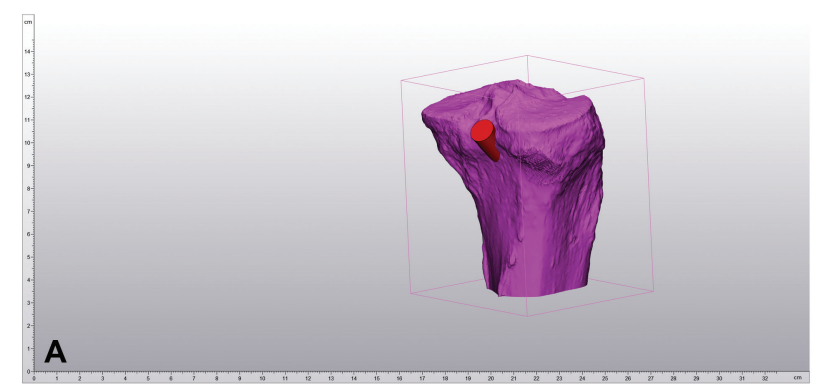

B
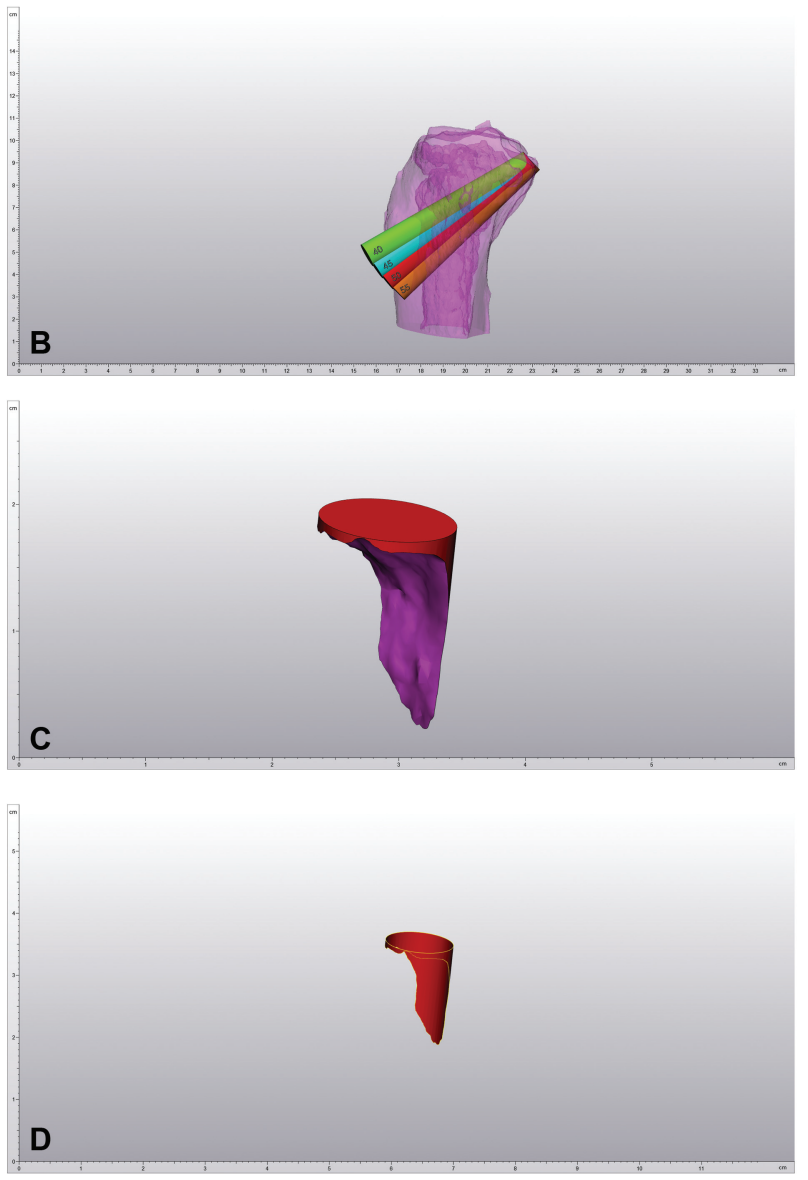

Fig. 2 Representative three-dimensional (3D) computed tomography $(C T)$ reconstruction with 50 degrees tibial tunnel present (A). All four potential tunnels visible (B). Tibial tunnel volume of the 50-degree tunnel not surrounded circumferentially by bone (C). Tibial tunnel surface area of the 50-degree tunnel not surrounded circumferentially by bone (D). circumferentially covered representing tibial cortical violation, was measured back to the point where the tibial tunnel circumferentially surrounded the tunnel. Additionally, the surface area and volume of this portion of the cylinder were determined ( - Fig. $\mathbf{2 C}$ and $\mathbf{D}$ ). The surface area and volume both in contact with tibial bone and that not in contact with the tibia were determined. Of note, the surface area attributable to the proximal and distal ends of the cylinder were not included in the measurements as this aspect of a graft would not be expected to be in contact with tibial bone.

Statistical analysis was performed using the SPSS software (version 27.0, IBM Corporation, Armonk, NY). Descriptive statistics were recorded. A paired samples $t$-test was used to determine whether the changing in tibia drilling angle from 55 degrees down to 40 degrees led to changes in stick-out length, volume, and surface area.

\section{Results}

The mean age of the CTs used in the study was 58.7 years (range 31-91). There were 6 female and 4 male CTs. There were 2 right knee CTs and 8 left knee CTs.

The means and standard deviations of the length of tibial tunnel not circumferentially surrounded by bone are listed in -Table 1. Of note, the length of tibial cortical violation, increases with increasing tibial drilling angle to a mean of $26.3 \mathrm{~mm}$ at 55 degrees. The mean exposed tibial tunnel length was significantly different comparing the 55-degree tunnel to each of the other tibial drilling angles $(p=0.000 ;$ - Table 2$)$.

The means and standard deviations of the surface area of tibial tunnel not circumferentially surrounded by bone are listed in - Table 1. Of note, the surface area increases with increasing tibial drilling angle to a mean of $372.2 \mathrm{~mm}$ at 55 degrees which represents $40 \%$ of the total surface area of the exposed cylinder length. The mean exposed surface area was significantly different comparing the 55-degree tunnel to each of the other tibial drilling angles $(p=0.000$; -Table 2).

The means and standard deviations of the volume of tibial tunnel not circumferentially surrounded by bone are listed in - Table 1. Of note, the volume increases with increasing tibial drilling angle to a mean of $840.8 \mathrm{~mm}$ at 55 degrees which represents $32 \%$ of the total volume of the cylinder

Table 1 Mean graft stick-out length, exposed volume, percent graft exposed, surface area exposed, and percent surface area exposed for each of the four listed tibia drilling angles

\begin{tabular}{|l|l|l|l|l|l|}
\hline Drilling angle & $\begin{array}{l}\text { Stick-out length } \\
\mathrm{mm}(\mathrm{mean}, \mathrm{SD})\end{array}$ & $\begin{array}{l}\text { Exposed volume } \\
\mathrm{mm}^{3} \text { (mean, SD) }\end{array}$ & $\begin{array}{l}\text { Exposed \% volume } \\
\text { (mean, SD) }\end{array}$ & $\begin{array}{l}\text { Surface area exposed } \\
\mathrm{mm}^{2} \text { (mean, SD) }\end{array}$ & $\begin{array}{l}\text { Exposed \% } \\
\text { surface area } \\
\text { (mean, SD) }\end{array}$ \\
\hline 55 degrees & $26.3(5.0)$ & $840.8(438.8)$ & $32(11)$ & $372.2(129.5)$ & $40(7)$ \\
\hline 50 degrees & $21.2(4.9)$ & $596.2(317.8)$ & $29(8)$ & $280.4(105.5)$ & $39(6)$ \\
\hline 45 degrees & $16.7(5.0)$ & $425.6(230.6)$ & $25(8)$ & $208.8(85.7)$ & $34(7)$ \\
\hline 40 degrees & $12.7(4.8)$ & $302.9(177.6)$ & $24(7)$ & $153.3(73.3)$ & $34(6)$ \\
\hline
\end{tabular}

Abbreviation: SD: standard deviation. 
PCL Tibial Tunnel Kim et al.

Table 2 Comparison of different drilling angles to the simulated 55-degree tibial tunnel for stick-out length, exposed volume, and exposed surface area

\begin{tabular}{|l|l|l|l|}
\hline Drilling angles & $\begin{array}{l}\text { Stick-out length } \mathrm{mm} \\
\text { (mean, SD) }\end{array}$ & $\begin{array}{l}\text { Exposed volume } \mathrm{mm}^{3} \\
\text { (mean, SD) }\end{array}$ & $\begin{array}{l}\text { Surface area exposed } \mathrm{mm}^{2} \\
\text { (mean, SD) }\end{array}$ \\
\hline 55-50 degrees & $5.1(1.3), p=0.000$ & $244.6(130.3), p=0.000$ & $91.8(28.5), p=0.000$ \\
\hline $55-45$ degrees & $9.6(1.9), p=0.000$ & $415.2(220.3), p=0.000$ & $163.3(47.4), p=0.000$ \\
\hline $55-40$ degrees & $13.6(2.3), p=0.000$ & $537.9(275.2), p=0.000$ & $218.9(59.6), p=0.000$ \\
\hline
\end{tabular}

Abbreviation: SD: standard deviation.

stick-out length. The mean exposed volume was significantly different comparing the 55-degree tunnel to each of the other tibial drilling angles ( $p=0.000$; - Table 2 ).

\section{Discussion}

We created transtibial tunnels using 3D CT modeling of standard PCL reconstruction techniques in attempts to better characterize the shape of the tunnel posteriorly. First, we found that there was consistent violation of the posterior cortex during tunnel creation. This resulted in an oblique aperture posteriorly, and an average length of $12.7 \mathrm{~mm}$ in which the tunnel was not fully circumferential with bone at the 40-degree tibia drilling angle which increased to $26.3 \mathrm{~mm}$ at a drilling angle of 55 degrees. As noted, decreasing the tunnel angle did decrease the amount of posterior cortex violation.

There have been extensive discussions regarding the anatomy and biomechanical function of the PCL. ${ }^{9-12}$ As descriptions of PCL anatomy grow and become more detailed, there have been increased efforts to create anatomic reconstructions to best simulate the native PCL. Femoral and tibial tunnel positioning have always been a critical aspect of anatomic reconstruction. Though traditionally there have been extensive discussion on femoral tunnel placement, there has also been increased interest in tibial insertional anatomy and tunnel positioning. ${ }^{10,15-17}$ The anatomy of the posterior tibia is quite unique, and has been described to consist of distinct anatomic regions including the tibial plateau, posterior cortex, and the posterior intercondylar fossa. This latter region represents a sloping central depression between the medial and lateral portions of the tibial plateau and has also been referred to as the PCL facet, fossa, or fovea. ${ }^{13}$ Moorman et al reported that the fibers of the PCL insert from the posterior cortex of the tibia to approximately $15 \mathrm{~mm}$ anteriorly along the PCL facet. This area represents the posterior half of the PCL facet. ${ }^{10}$ Therefore, to achieve an anatomic tibial socket, a guidewire should be placed central to this footprint, which is $7 \mathrm{~mm}$ anterior to the posterior cortex. ${ }^{10}$ Similarly, Lee et al reported that the PCL insertion was in the posterior $48 \%$ of the PCL facet. In this anatomic study, they report the same concern for tibial tunnel posterior cortex violation and explain the importance of understanding the unique anatomy of the posterior tibia. When attempting an anatomic tunnel centered on the PCL tibial footprint, they found that the tibial guide must be limited to
52 degrees to prevent posterior cortex violation distal on the tibia to the drill exit point. ${ }^{14}$ Similarly, in the current study, it was found that a simulated angle of 55 degrees produced tibial tunnels with consistent violation of the posterior cortex. On average, this defect measured $26.3 \mathrm{~mm}$ in length and a traversing graft would have to be longer than this before being completely surrounded by bone. The current study differs from Lee et al in that there was also consistent compromise of the posterior cortex at lesser angles up to 40 degrees. As hypothesized, the amount of posterior cortex violation decreased with decreasing angles. However, Lee et al also chose a $10-\mathrm{mm}$ tibial tunnel in their study, whereas the current study simulated an 11-mm tunnel. This could certainly account for some of the differences in the results.

This and other studies demonstrate that lowering the tibial tunnel drilling angle helps decrease the obliquity of the posterior tibial tunnel exit and therefore the amount of uncovered graft in the posterior tibial tunnel. Often PCL reconstructions are performed with concomitant ACL reconstruction in multiligament injured knees. If two tunnels are drilled for both cruciate reconstructions, then the PCL reconstruction tunnel is typically drilled distal to the ACL reconstruction tunnel. ${ }^{18,19}$ In this setting it would be challenging to decrease the PCL tibial drilling angle as the more proximal ACL tunnel may then converge within the tibia.

Johannsen et al reported radiographic landmarks for tunnel positioning as well. ${ }^{20}$ In this detailed study, they included radiographic landmarks for the PCL center as well as the two bundles separately. They reported that on the lateral view, the anterolateral bundle center was $8.4 \pm 1.8 \mathrm{~mm}$, the posteromedial bundle was $2.5 \pm 1.5 \mathrm{~mm}$, and the PCL center was $5.5 \pm 1.7 \mathrm{~mm}$ from the so-called champagne glass drop-off of the posterior cortex. These measurements appear similar to previous anatomic descriptions of PCL insertional anatomy. They once again highlight the close proximity of the PCL to the champagne glass dropoff and the real possibility of posterior cortex violation.

Nonanatomic tunnel placement would risk a poor functioning graft and theoretically increase the risk for failure. With respect to the location of the posterior tibial tunnel, too anterior of a tunnel could risk injury to the articular surface and posterior horns of the menisci. A tunnel placed too posterior could risk injury to the neurovascular bundle, ${ }^{21}$ and also increase the "killer-turn." On the other hand, this study found that an anatomically placed tunnel using standard transtibial techniques risks compromising the posterior 
cortex of the tibia, thus producing a tunnel not fully circumferential with bone. This should be concerning when considering the importance of graft to bone healing in some reconstruction procedure techniques. This is most relevant to arthroscopic PCL reconstruction techniques that involve placing a graft's tibial side, either soft tissue or bone plug, into a posterior tibial socket. ${ }^{22-26}$ For example, the bone plug on an Achilles allograft, as is customary for arthroscopic inlay reconstruction, would not be fully seated in bone at an average of $12.7 \mathrm{~mm}$ of bone plug length and would not be fully surrounded by bone. Additionally, a graft could hinge posteriorly if there is insufficient bone holding it in the socket against the posterior proximal tibia.

This study has several limitations. First, it is not a clinical or cadaveric study. CT scans of knees from real patients were utilized to create 3D models, and transtibial tunnels were simulated in a manner resembling standard PCL reconstructions techniques. This was done to allow simulation of various tunnel angles, and to allow for accurate measurements of cortex violation and exposed grafts. In addition, this study tried best to simulate only one of the many techniques of PCL reconstruction, and the findings may not directly apply to some surgeons. We decided to simulate an 11-mm tunnel, though some may prefer a different size graft or tunnel. We also created a transtibial tunnel, while some surgeons may prefer sockets or utilize an inlay technique. Nevertheless, we feel the findings of the study still bring light to the unique anatomy of the posterior tibia after a socket or tunnel is created. Lastly, this is not a clinical or biomechanical study and therefore does not intend to determine any such significance. Though the findings of the 3D modeling are interesting, we cannot comment on its effect on graft stability, healing, or outcomes as any clinical effects on healing have not yet been described.

In conclusion, anatomic tibial tunnel creation using standard transtibial PCL reconstruction techniques consistently risks posterior tibial cortex violation and creation of an oblique aperture posteriorly. This risk is decreased with decreasing the angle of the tibial tunnel, though the posterior cortex is still compromised with angles as low as 40 degrees. With posterior cortex violation, the surgeon should be aware that a graft within the tunnel or socket posteriorly may not be fully in contact with bone thus decreasing the healing interface of the graft within the socket. This is especially relevant with inlay and socket techniques.

\section{Ethical Approval}

This study received its institutional review board (IRB) approval at Saint Louis University 1/17/2018, Protocol \#28746.

\section{Conflict of Interest \\ None declared.}

\section{Acknowledgment}

The authors would like to thank Heidi Israel, PhD, for her assistance with the statistics for the study.

\section{References}

1 Deehan DJ, Cawston TE. The biology of integration of the anterior cruciate ligament. J Bone Joint Surg Br 2005;87(07):889-895

2 Rodeo SA, Arnoczky SP, Torzilli PA, Hidaka C, Warren RF. Tendonhealing in a bone tunnel. A biomechanical and histological study in the dog. J Bone Joint Surg Am 1993;75(12):1795-1803

3 Rodeo SA, Kawamura S, Kim HJ, Dynybil C, Ying L. Tendon healing in a bone tunnel differs at the tunnel entrance versus the tunnel exit: an effect of graft-tunnel motion? Am J Sports Med 2006;34 (11): $1790-1800$

4 Greis PE, Burks RT, Bachus K, Luker MG. The influence of tendon length and fit on the strength of a tendon-bone tunnel complex. A biomechanical and histologic study in the dog. Am J Sports Med 2001;29(04):493-497

5 Mariscalco MW, Magnussen RA, Mitchell J, et al. How much hamstring graft needs to be in the femoral tunnel? A MOON cohort study. Eur Orthop Traumatol 2015;6(01):9-13

6 Qi L, Chang C, Jian L, Xin T, Gang Z. Effect of varying the length of soft-tissue grafts in the tibial tunnel in a canine anterior cruciate ligament reconstruction model. Arthroscopy 2011;27(06): 825-833

7 Yamazaki S, Yasuda K, Tomita F, Minami A, Tohyama H. The effect of intraosseous graft length on tendon-bone healing in anterior cruciate ligament reconstruction using flexor tendon. Knee Surg Sports Traumatol Arthrosc 2006;14(11):1086-1093

8 Zantop T, Ferretti M, Bell KM, Brucker PU, Gilbertson L, Fu FH. Effect of tunnel-graft length on the biomechanics of anterior cruciate ligament-reconstructed knees: intra-articular study in a goat model. Am J Sports Med 2008;36(11):2158-2166

9 Edwards A, Bull AM, Amis AA. The attachments of the fiber bundles of the posterior cruciate ligament: an anatomic study. Arthroscopy 2007;23(03):284-290

10 Moorman CT III, Murphy Zane MS, Bansai S, et al. Tibial insertion of the posterior cruciate ligament: a sagittal plane analysis using gross, histologic, and radiographic methods. Arthroscopy 2008; 24(03):269-275

11 Tajima G, Nozaki M, Iriuchishima T, et al. Morphology of the tibial insertion of the posterior cruciate ligament. J Bone Joint Surg Am 2009;91(04):859-866

12 Takahashi M, Doi M, Abe M, Suzuki D, Nagano A. Anatomical study of the femoral and tibial insertions of the anteromedial and posterolateral bundles of human anterior cruciate ligament. Am J Sports Med 2006;34(05):787-792

13 Girgis FG, Marshall JL, Monajem A. The cruciate ligaments of the knee joint. Anatomical, functional and experimental analysis. Clin Orthop Relat Res 1975;(106):216-231

14 Lee YS, Ra HJ, Ahn JH, Ha JK, Kim JG. Posterior cruciate ligament tibial insertion anatomy and implications for tibial tunnel placement. Arthroscopy 2011;27(02):182-187

15 Inderster A, Benedetto KP, Klestil T, Künzel KH, Gaber O. Fiber orientation of posterior cruciate ligament: an experimental morphological and functional study, Part 2. Clin Anat 1995;8(05): 315-322

16 Racanelli JA, Drez D Jr. Posterior cruciate ligament tibial attachment anatomy and radiographic landmarks for tibial tunnel placement in PCL reconstruction. Arthroscopy 1994;10(05): 546-549

17 Sheps DM, Otto D, Fernhout M. The anatomic characteristics of the tibial insertion of the posterior cruciate ligament. Arthroscopy $2005 ; 21(07): 820-825$

18 Fanelli GC, Orcutt DR, Edson CJ. The multiple-ligament injured knee: evaluation, treatment, and results. Arthroscopy 2005;21 (04):471-486

19 Moatshe G, Slette EL, Engebretsen L, LaPrade RF. Intertunnel relationships in the tibia during reconstruction of multiple knee ligaments: how to avoid tunnel convergence. Am J Sports Med 2016;44(11):2864-2869 
PCL Tibial Tunnel Kim et al.

20 Johannsen AM, Anderson CJ, Wijdicks CA, Engebretsen L, LaPrade RF. Radiographic landmarks for tunnel positioning in posterior cruciate ligament reconstructions. Am J Sports Med 2013;41(01):35-42

21 Makino A, Costa-Paz M, Aponte-Tinao L, Ayerza MA, Muscolo DL. Popliteal artery laceration during arthroscopic posterior cruciate ligament reconstruction. Arthroscopy 2005;21(11):1396

22 Kim SJ, Choi CH, Kim HS. Arthroscopic posterior cruciate ligament tibial inlay reconstruction. Arthroscopy 2004;20(Suppl 2):149-154

23 Kim SJ, Park IS. Arthroscopic reconstruction of the posterior cruciate ligament using tibial-inlay and double-bundle technique. Arthroscopy 2005;21(10):1271
24 Mariani PP, Margheritini F. Full arthroscopic inlay reconstruction of posterior cruciate ligament. Knee Surg Sports Traumatol Arthrosc 2006;14(11):1038-1044

25 Osti M, Hierzer D, Seibert FJ, Benedetto KP. The arthroscopic allinside tibial-inlay reconstruction of the posterior cruciate ligament: medium-term functional results and complication rate. J Knee Surg 2017;30(03):238-243

26 Salata MJ, Sekiya JK. Arthroscopic posterior cruciate ligament tibial inlay reconstruction: a surgical technique that may influence rehabilitation. Sports Health 2011;3(01):52-58 